\title{
Design Optimization for Cryo-Tomography and Single Particle Data Acquisition and Analysis
}

\author{
B.L. Armbruster*, J. Brink*, R. Danev**, T. Isabell*, M. Kawasaki*, S. Motoki*** and K. \\ Nagayama** \\ *JEOL USA Inc., Peabody MA 01960 \\ **Okazaki Institute for Integrative Bioscience, National Institutes of Natural Sciences, Dept. \\ Physiological Sciences, The Graduate University of Advanced Studies, Higashiyama 5-1, \\ Myodaijicho, Okazaki 444-8787 Aichi, Japan \\ ***JEOL Ltd., 1-2 Musashino 3-chome, Akishima Tokyo 196-8558, Japan
}

Exploring the new frontier of cell biology and structural biochemistry requires single particle analysis and cryo-tomography to image the complete proteome of cells in a near-native solution state. To attain this goal, the quantity and quality of data collection must improve. Most laboratories are adopting the advanced technology of high performance cryoelectron microscopes equipped with a range of hardware and software modifications to enhance contrast and resolution [1]. Compared with the sample constraints of x-ray crystallography and nuclear magnetic resonance, in-situ electron microscopy of biological nanomachines does not require crystalline samples in non-"native" buffers to obtain high resolution single particle data, as evidenced by the $4.5 \AA$ resolution structure of the epsilon 15 capsid protein gene protein 7 [2]. Spherical aberration correction has made sub-angstrom resolution imaging and EELS mapping of inorganic materials a reality [3], and significant improvements should be possible for biological specimens as indicated by the first aberration-corrected cryoexperiments of Evans et al. [4] on organic specimens.

Automation of data collection is essential to assign heterogeneous, flexible particles into classes of homogeneous conformations before image reconstruction. Recent software developments in automated cryo-EM low dose operation for single particle analysis include the JEOL Automated Data Acquisition System (JADAS) [5]. Designed for use with commercially-available cryogrids, JADAS' multiscale grid-searching algorithm performs a low-magnification global search of grid squares followed by a local search in selected grid squares to find ice of a suitable thickness. Microscope and camera operations are saved in the form of customizable, reusable "recipes" which can be modified according to the parameters of the experiment. Images are recorded at a user-defined dose, defocus and magnification to generate data for sub-nanometer resolution reconstructions.

Cryo-tomography (CT) can provide the cellular context in which high-resolution reconstructions obtained by single particle approaches can be placed. Or, it allows for the study of cellular structures in the native state without potential artifacts introduced by fixation and staining procedures. SerialEM [6] has been successfully applied to many JEOL installations and the software provides for extensive control over the microscope to enable CT under low-dose conditions. Key features include complete control of either in-column or post-column energy filters, a powerful scripting environment supporting conical tomography [7], as well as very extensive support for montaging using either side-mounted or bottommounted cameras. Processing and visualizing the tomographic data is accomplished within IMOD and Chimera, respectively [8,9].

One technique to improve contrast for single particle analysis and cryo-tomographic data collection involves the use of phase plates. Phase contrast light microscopy has long been an 
indispensible tool for live-cell imaging, but this effective technique has only recently been successfully applied to transmission electron microscopy with thin film phase plates. A Zernike phase plate consists of an amorphous carbon film of defined thickness with a small central hole. The zero-order beam traverses the central hole while electrons traversing the thin film are phase-shifted by $\pi / 2$. Starting with Boersch's concepts [10], the research of Nagayama [11,12] has illustrated the potential for phase contrast TEM imaging of low contrast, vitrified macromolecules. The 3-D model of GroEL [13] at $12 \AA$ resolution based on phase-contrast images required $30 \%$ fewer particles compared to a conventional image set, results especially critical for scientists examining heterogeneous samples, specimens available at low concentration or structures with low symmetry.

Microscope column designs can be adapted at various levels for phase plate applications. At a basic level for routine low-dose imaging and contrast enhancement, modifications to existing TEM columns could include the addition of an objective aperture holder engineered to hold a phase plate [14], a 25-hole phase plate grid made of molybdenum [15] to maximize the number of phase plates available plus control software to accurately center the phase plate. A phase plate holder should be designed with piezo drives for precision positioning and continuous heating of the phase plate to $\sim 200^{\circ} \mathrm{C}$ to minimize charging and contamination.

The optimal design for phase contrast cryo-electron tomography demands careful consideration of all optical elements to maximize image contrast, and features necessary in a dedicated column configuration have been proposed by Danev et al. [16]. Their ideal column would include a field-emission gun, a three lens condenser system with independent adjustment for each lens, and an objective focal length of $5 \mathrm{~mm}$ or larger to accommodate the phase plate holder plus a room-temperature aperture to serve as a heat shield. The small size of the central hole of the phase plate requires accurate software control to center parallel illumination in all low dose modes and tilt angles. Zero-loss energy filtering roughly doubles the signal-to-noise ratio [17] and is essential for removing inelastic scattering when imaging thick samples. The realization of this design should extend the practical examination of the proteome to include nanomachines as small as $100 \mathrm{kDa}$.

References

[1] B.L. Armbruster et al., Microsc. Microanal. 14 (Suppl 2) (2008) 1072.

[2] W. Jiang et al., Nature 451 (2008) 1130.

[3] E. Okunishi et al., Microsc. Microanal. 14 (Suppl 2) (2007) 1372.

[4] J.E. Evans et al., Ultramicroscopy 108 (2008) 1636.

[5] J. Zhang et al., J. Struct. Biol. 165 (2009) 1.

[6] D.N. Mastronarde, J. Struct. Biol. 152 (2005) 36.

[7] S. Lanzavecchia et al., J. Struct. Biol. 149 (2005) 87.

[8] J.R. Kremer et al., J. Struct. Biol. 116 (1996) 71.

[9] E.F. Pettersen et al., J. Comput. Chem. 25 (2004) 1605.

[10] H. Boersch, Z. Naturforsch. 2a (1947) 615.

[11] K. Nagayama and R. Danev, Phil. Trans. Roy. Soc. B (2008) 2153.

[12] M. Yamaguchi et al., J. Struct. Biol. 162 (2008) 271.

[13] R. Danev and K. Nagayama, J. Struct. Biol. 161 (2008) 211.

[14] M. Marko, Microsc. Microanal. 13 (Suppl 2) (2007) 1212.

[15] K. Nagayama, Eur. Biophys. J. 37 (2008) 345.

[16] R. Danev et al., Ultramicroscopy on line Dec. 11, 2008.

[17] R. Grimm et al., J. Microsc. 190 (1998) 339. 